\title{
Dynamical Influences of the Last Magellanic Interaction on the Magellanic Clouds
}

\author{
Kenji Bekki ${ }^{\mathrm{A}, \mathrm{C}}$ and Masashi Chiba ${ }^{\mathrm{B}}$ \\ A School of Physics, University of New South Wales, Sydney NSW 2052, Australia. \\ B Astronomical Institute, Tohoku University, Sendai 980-8578, Japan. \\ C Corresponding author. Email: bekki@phys.unsw.edu.au
}

Received 2006 October 8, accepted 2007 February 19

\begin{abstract}
We investigate the present distributions of gas and young stars in the Large and Small Magellanic Clouds (LMC and SMC) based on fully self-consistent numerical simulations of the Clouds for the last $\sim 0.8$ Gyr. Our principal results, which can be tested against observations, are as follows. The last dynamical and hydrodynamical interaction between the Clouds about $\sim 0.2 \mathrm{Gyr}$ ago can form the apparently off-center bar and peculiar Hi spirals of the LMC. The present spatial distributions of young stars with ages less than $\sim 20$ Myr in the LMC can be significantly asymmetric and clumpy owing to the interaction. A small but nonnegligible fraction of stellar and gaseous components can be transferred from the SMC into the LMC during the interaction to form diffuse halo components around the LMC. The burst of star formation in the SMC can be synchronized with that of the LMC about $0.2 \mathrm{Gyr}$ ago in some models. New stars can form from gas in the SMC's tidal tails, one of which can be observed as the Magellanic Bridge (MB). The metallicity distribution function of new stars in the MB has a peak of $[\mathrm{Fe} / \mathrm{H}] \sim-0.8$, which is significantly smaller than the stellar metallicity of the SMC. Based on these results, we discuss the origin of 30 Doradus, the southern molecular ridge of the LMC, the globular cluster ESO 121-SC03, metal-poor inter-Cloud stars within the MB, and giant Hi holes of the LMC.
\end{abstract}

Keywords: galaxies: Magellanic Clouds — galaxies: star clusters — galaxies: stellar content — galaxies: interactions

\section{Introduction}

The Large Magellanic Cloud (LMC) morphologically classified as an irregular galaxy (Ir III-IV) is observed to have a prominent off-center bar embedded within its flat disk component (e.g. de Vaucouleurs \& Freeman 1972; van den Bergh 2000). Recent observational studies based on the Deep Near-Infrared Southern Sky Survey (DENIS) and Two Micron All-Sky Survey (2MASS) have revealed that the LMC has the off-center stellar bar in a significantly elongated stellar disk (e.g. van der Marel 2001). Influences of the off-center bar on the evolution of the LMC (e.g. the recent star formation history) have been discussed by several authors (e.g. Gardiner et al. 1998).

Such asymmetric structures seen in the LMC's stellar components have been revealed also in the gaseous ones by multibeam Hi survey of the LMC (e.g. Staveley-Smith et al. 2003) and by the first CO survey with NANTEN (Fukui et al. 1999). The 'molecular ridge' observed in the southern part of the LMC has a peculiar distribution of molecular clouds and is considered to be a possible formation cite of new stars and clusters (Fukui et al. 1999; Kawamura et al. 2007). Remarkably asymmetric and patchy distributions of star-forming complexes and young populous clusters are also observed within the stellar disk of the LMC (van den Bergh 2000). Although the observed asymmetric distributions in variously different populations (e.g. carbon stars, HI, and star-forming regions) are suggested to provide valuable information on the LMC's evolution interacting with the Small Magellanic Cloud (SMC) and the Galaxy (e.g. Westerlund 1997), it is unclear when and how these asymmetric structures were formed in the dynamical history of the LMC.

The purpose of this paper is thus to demonstrate, for the first time, that the last dynamical interaction between the LMC and the SMC about $0.2 \mathrm{Gyr}$ ago can be responsible for the formation of the off-center bar, peculiar spirals, and asymmetrically distributed young stars in the LMC. Although previous studies already pointed out the importance of the Magellanic collision in the formation of the LMC's young star clusters (e.g. Fujimoto \& Kumai 1997; Bekki et al. 2004a) and a peculiar structure seen in the periphery of the LMC (e.g. Kunkel et al. 1997), the present study first explains the origin of asymmetric structures in both stellar and gaseous components of the LMC in a self-consistent manner.

Although our main focus is on the two-dimensional distributions of gas and stars in the LMC, we also investigate the SMC's stellar and gaseous evolution that has not been investigated by previous numerical studies (e.g. Yoshizawa \& Noguchi 2003). We particularly discuss the origin of young, metal-poor stars observed in the interCloud regions such as the Magellanic Bridge (MB, e.g. 
Rolleston et al. 1999). We here focus on the essence of the physical mechanisms (1) for the formation of asymmetric structure in the LMC and (2) for star formation in the MB and accordingly will make more quantitative comparison of the simulation results with the latest observations in our forthcoming papers.

The plan of the paper is as follows. In Section 2 we describe our numerical models for dynamical evolution of the LMC and the SMC interaction with each other and with the Galaxy. In Section 3 we present the numerical results on structural properties and recent stat formation histories of the LMC and the SMC. In Section 4 we discuss the derived numerical results in several different contexts of the LMC's and the SMC's evolution, such as the origin of 30 Doradus in the LMC. We summarise our conclusions in Section 5.

\section{The Model}

Since fundamental methods and techniques of numerical simulations on the evolution of the Clouds are given in our previous paper (Bekki \& Chiba 2005, hereafter BC05), we briefly describe them here. We investigate the dynamical and hydrodynamical evolution and star formation histories of the Clouds from $0.8 \mathrm{Gyr}$ ago $(T=-0.8 \mathrm{Gyr})$ to the present $(T=0)$, by using chemodynamical simulations in which both the LMC and the SMC are modeled as selfgravitating, barred and disk systems, composed initially of dark matter halos, stars ('old stars'), and gas. The total number of particles used for a galaxy is 120000 for all models in the present study. We first determine the most plausible and realistic orbits of the Clouds and then investigate the evolution of the LMC using fully self-consistent GRAPE-SPH simulations (Bekki \& Chiba 2006). In determining the orbits, we adopt the same numerical method as those in previous studies (Murai \& Fujimoto 1980; Gardiner \& Noguchi 1996, hereafter GN96), in which the equations of motion of the Clouds are integrated backward in time, from the present epoch until $\sim 0.8 \mathrm{Gyr}$ ago. The orbital evolution of the Clouds in different models is given in $\mathrm{BC} 05$.

The two Clouds are modeled as self-gravitating disk galaxies (with total masses of $M_{\mathrm{t}}$ ) embedded by massive dark matter halos (GN96, BC05). The mass fraction of baryonic components (i.e. stellar and gaseous ones) in a galaxy $\left(f_{\mathrm{b}}\right)$ is set to be 0.3 both for the LMC and for the SMC in the present study. The Hi diameters of gasrich galaxies are generally observed to be larger than their optical disks (e.g. Broeils \& van Woerden 1994). Guided by these observations, the gas disks $\left(R_{\mathrm{g}}\right)$ of the Clouds are assumed to be at least 1.5 times larger than their stellar disks $\left(R_{\mathrm{S}}\right)$ in the present models. The initial $R_{\mathrm{S}}$ is $7.5 \mathrm{kpc}$ for the LMC, which means that both the stellar and gaseous disks are well within the tidal radius of the LMC if $R_{\mathrm{g}} / R_{\mathrm{S}}<15 \mathrm{kpc}$. The initial $R_{\mathrm{S}}$ is $5.0 \mathrm{kpc}$ for the $\mathrm{SMC}$ which is similar to that adopted in GN96. The gas mass fraction $\left(f_{\mathrm{g}}\right)$ is set to be 0.1 both for the LMC and for the SMC. The gas is converted into new field stars ('new stars') according to the Schmidt law with the observed threshold
Table 1. Model parameters

\begin{tabular}{lllll}
\hline Galaxy & \multicolumn{1}{c}{$M_{\mathrm{t}}$} & $f_{\mathrm{b}}$ & $f_{\mathrm{g}}$ & $R_{\mathrm{s}}$ \\
\hline LMC & $2 \times 10^{10} \mathrm{M}_{\odot}$ & 0.3 & 0.1 & $7.5 \mathrm{kpc}$ \\
SMC & $3 \times 10^{9} \mathrm{M}_{\odot}$ & 0.3 & 0.1 & $5.0 \mathrm{kpc}$ \\
\hline
\end{tabular}

gas density (Kennicutt 1998). The adopted parameter values (e.g. $f_{\mathrm{g}}$ ) are summarized in the Table 1 .

The method to set up initial disks with stellar bars, which are quite reasonable for the LMC and the SMC about $\sim 1$ Gyr ago (BC05), is described as follows. We first dynamically relax the exponential disk composed purely of stars embedded in the dark matter halo and thereby form a stellar bar (by invoking the global bar instability). Then we add an exponential gas disk to the relaxed disk with the bar and give kinematical information (e.g. circular velocities etc.) to each of the gas particle. Thus a disk with a stellar bar and an exponential gas disk embedded in a massive dark matter halo can be set up for the Clouds.

Although we obtain the results of models with different masses (thus orbits) of the LMC $\left(M_{\mathrm{L}}\right)$ and the SMC $\left(M_{\mathrm{S}}\right)$ for a reasonable range of their initial masses, we show the results of the standard model with $M_{\mathrm{L}}=2.0 \times 10^{10} \mathrm{M}_{\odot}$ and $M_{\mathrm{s}}=3.0 \times 10^{9} \mathrm{M}_{\odot}$. This is firstly because the results of this model can be compared with previous simulations with the same model parameters (e.g. GN96) and secondly because this model shows typical behaviors of off-center bar formation. $R_{\mathrm{g}} / R_{\mathrm{S}}$ is set to be 1.5 for the LMC and 2.0 for the SMC in models. The models with $R_{\mathrm{g}} / R_{\mathrm{S}}=1$ do not show any significant differences in the spatial distributions between gas and stars in the LMC. We do not intend to show the result of these models, mainly because they are not so consistent with observations.

We use the same coordinate system $(X, Y, Z)$ (in units of kpc) as those used in GN96 and BC05. The adopted current positions are $(-1.0,-40.8,-26.8)$ for the LMC and $(13.6,-34.3,-39.8)$ for the SMC and the adopted current Galactocentric radial velocity of the LMC (SMC) is $80(7) \mathrm{km} \mathrm{s}^{-1}$. Current velocities of the LMC and the SMC in the Galactic $(U, V, W)$ coordinate are assumed to be $(-5,-225,194)$ and $(40,-185,171)$ in units of $\mathrm{km} \mathrm{s}^{-1}$, respectively. The model for the disk configuration (e.g. inclination angles) of the LMC (SMC) is exactly the same as that in BC05 (GN96). The initial inclinations of the Clouds with respect to the above coordinate are set to be the same as those adopted in GN96 (SMC) and BC05 (LMC).

Although the adopted orbital model is broadly consistent with previous ones that successfully explained many observational results (GN96, BC05), the orbital model of the SMC is not so consistent with the recent observation by Kallivayalil, van der Marel \& Alcock (2006). As shown in Figure 13 of Kallivayalil et al. (2006), the LMC and the SMC strongly interact with each other about $0.2 \mathrm{Gyr}$, if their proper motion data are considered in orbital calculations. We thus suggest that as long as the last $1 \mathrm{Gyr}$ 
evolution is concerned, the models with orbital parameters suggested by Kallivayalil et al. (2006) would show similar results to those in the present paper.

We derive the two-dimensional (2D) distributions of the $B$-band surface brightness $\left(\mu_{\mathrm{B}}\right)$ in the LMC's stellar disk from the simulated mass distributions of its old and new stars by assuming that the stellar mass-to-light-ratio $\left(M / L_{\mathrm{B}}\right)$ is 4.0 for old stars and 0.16 for new ones (Vazdekis et al. 1996). In order to show more clearly the simulation results in some figures, we make the following coordinate transformation for the projected mass distributions to see more clearly the face-on morphology of the LMC: Firstly the LMC disk is rotated by some degrees in the $x-z$ plane (i.e. about the $y$-axis) such that the major axis of the bar is coincident with the $x$-axis, then the disk is rotated by 180 degrees about the $z$-axis. We investigate (1) the model without SMC ('SMC-less' model) and (2) the model in which the LMC interacts neither with the Galaxy nor with the SMC ('isolated' model) so that we can more clearly understand the roles of SMC in the LMC evolution. We also investigate the evolution of the Clouds from $T=0 \mathrm{Gyr}$ to $T=+6 \mathrm{Gyr}$ (i.e. the next $6 \mathrm{Gyr}$ ) to discuss the final dynamical fate of the Clouds.

Although we investigated many models with different orbits depending on the adopted initial velocities that are consistent with observations on proper motion of the Clouds, we here show the results of the standard model, because this model enables us to describe clearly the essential roles of the last Magellanic interaction in structural properties and recent star formation histories of the Clouds in a convincing way. We plan to discuss how numerical results depend on a number of parameters of the Magellanic system (e.g. orbits, masses of the LMC and the SMC, and parameters of star formation) in our forthcoming papers. Since we mainly focus on 2D distributions of gas and stars in the present Clouds, we do not intend to describe dynamical evolution of the Clouds in the present study. The details of dynamical evolution (e.g. morphological evolution of the LMC) of the Magellanic system have already been given in $\mathrm{BC} 05$.

Figure 1 shows the orbital evolution of the LMC and the SMC with respect to the Galaxy for the last $\sim 0.8 \mathrm{Gyr}$ in the standard model. Although the present model includes dynamical friction between the LMC and the SMC (owing to the fully self-consistent model of the Magellanic system), the orbital evolution is quite similar to that in previous models without dynamical friction between the Clouds (e.g. GN95; BC05). Because of the small pericenter distance $(8 \mathrm{kpc})$ in the LMC-SMC orbit, the outer parts of the gas disks of the Clouds collide with each other to form peculiar gaseous structures in the Clouds for this model. The pericenter distance of the LMC-SMC orbit, which is a key parameter for the final 2D-distributions of gas and stars in the Clouds, strongly depends on the initial velocities of the Clouds in the present models. Thus it should be stressed here that it depends on the adopted initial velocities of Clouds whether (and when) the Clouds collide with each other in the present models.

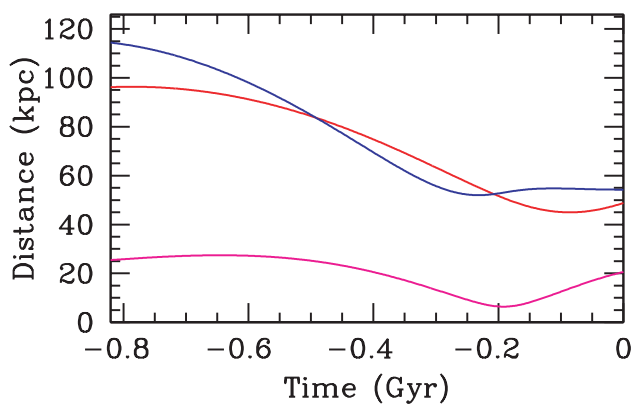

Figure 1 Time evolution of distance between the LMC and the SMC (magenta), the LMC and the Galaxy (red), and the SMC and the Galaxy (blue) for the last $0.8 \mathrm{Gyr}$ in the standard model. Note that the LMC-SMC distance becomes minimum $(8 \mathrm{kpc})$ about 0.2 Gyr ago.

The pericenter distance of $8 \mathrm{kpc}$ in the LMC-SMC orbit shown in Figure 1 about $0.2 \mathrm{Gyr}$ ago means that the tidal force from the LMC is about 20 times stronger than that from the Galaxy for the SMC. This thus means that the SMC can be much more strongly influenced by the LMC-SMC interaction than the SMC-Galaxy one. This LMC-SMC interaction can also significantly influence stellar and gaseous evolution of the LMC and thus its recent star formation history. We thus discuss how the tidal interaction controls the final $(T=0)$ distributions of gas and young stars in the Clouds.

In the present study, we show the results of the most important model that should be compared with the corresponding observations. Although the results can depend strongly on the orbits of the LMC and SMC, for which there are observational uncertainties. For example, if we adopt the model with a much larger pericenter distance ( $>20 \mathrm{kpc}$ ) between the LMC and the SMC, around 0.2 Gyr ago, the asymmetric appearance of the LMC becomes less remarkable and the total amount of gas and stars stripped from the SMC also becomes small. We do not intend to discuss the models with variously different parameters in the present study, mainly because the results of most of these models are not so consistent with observations. It should be noted here that the 'isolated' LMC model without interaction (of the LMC) with the Galaxy and the SMC does not show any remarkable asymmetric spiral arms seen in the model described in the present study (e.g. Figure 2). This confirms that the simulated asymmetric spiral arms are not due to numerical artifacts of initial gaseous disks adopted in the present study.

\section{Results}

\subsection{The $L M C$}

Figure 2 shows the time evolution of the gaseous distribution of the LMC's disk interacting with the SMC and the Galaxy. Owing to the combined tidal fields of the SMC and the Galaxy, the LMC's outer gas disk is strongly disturbed to form peculiar gaseous arms in the disk $(T=-0.27 \mathrm{Gyr})$. The gas disk also hydrodynamically interacts with the SMC's gas disk during the pericenter 


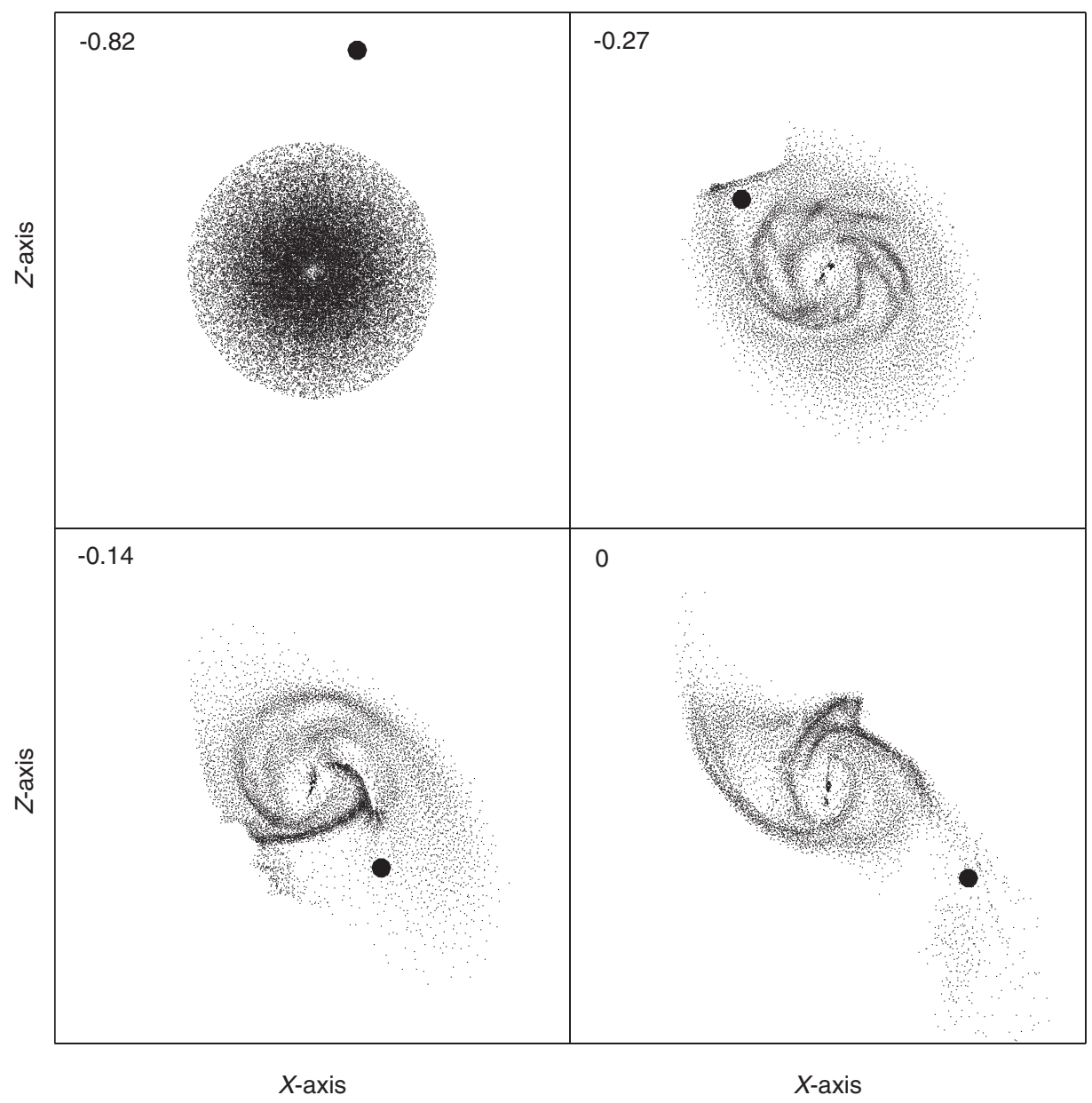

Figure 2 Distributions of the LMC's gas particles projected onto the $x-z$ plane at different epochs. For convenience, the center of each frame is set to be coincident with the center of the LMC. Each frame measures $45 \mathrm{kpc}$ and the number shows in the upper left corner of each frame represents the time $T$ in units of Gyr. The gaseous evolution from $0.82 \mathrm{Gyr}$ ago $(T=-0.82)$ to the present $(T=0)$ is shown in these four frames. For clarity, gas particles of the SMC are not shown and the center of the SMC is shown by a big dot. Total number of gas particles dramatically decreases owing to gas consumption by star formation as time passes by. Most gas in the central $3 \mathrm{kpc}$ of the LMC's disk is converted into new stars to form a stellar bar.

passage of the SMC $(T=-0.14 \mathrm{Gyr})$ so that asymmetric gaseous arms with high-densities can be developed in the disk. A small fraction of the LMC's gas can be stripped and dispersed into the region between the LMC and the SMC (i.e. inter-Cloud region) during tidal interaction to form diffuse gaseous components in the inter-Cloud region. An elongated gas disk with a number of asymmetric, peculiar spiral arms is finally formed without the stellar disk being severely disturbed ( $T=0 \mathrm{Gyr})$.

Figure 3 shows the 2D distributions of $\mu_{\mathrm{B}}$ of the LMC projected onto the $x-z$ plane at $T=0 \mathrm{Gyr}$ for the standard model and the SMC-less one. Although the tidal interaction between the LMC and the Galaxy can form an elliptical stellar disk, no off-center bar can be formed in the SMC-less model. The standard model, on the other hand, shows a clear asymmetric distribution in the outer disk with respect to the central bar and, accordingly, the central bar appears to be largely shifted from the center of the disk. The results of these two models clearly demonstrate that the Magellanic interaction can be responsible for the formation of the apparently off-center bar embedded within the LMC's elliptic stellar disk. The result of the SMC-less model is quantitatively consistent with previous models without SMC evolution (e.g. BC05).

It should be stressed that the simulated asymmetric 2D distributions are due to dynamical interaction between the LMC and the SMC (and the Galaxy): they are not due to the collision between the two gas disks. The contribution of old stars is $90 \%$ in mass and $26 \%$ in light owing to the adopted $M / L=4.0$ for old stars. This means that the derived asymmetric 2D distributions are due largely to young stars formed in the asymmetric gas.

As Figure 4 reveals, showing the 2D distribution of the surface gas density $\left(\mu_{\mathrm{g}}\right)$ of the standard model, the Magellanic interaction results in the formation of a significantly asymmetric gas distribution with higher gas densities in the left side of the stellar bar. Two gaseous arms appear to emerge from above the left end of the bar with the one extending to the direction of $(X, Z) \approx(-7,0) \mathrm{kpc}$ and the other extending to the direction of $(X, Z) \approx(-2,-7)$ $\mathrm{kpc}$. The latter gaseous arm is furthermore connected to the Magellanic gaseous bridge in the inter-Magellanic region. 

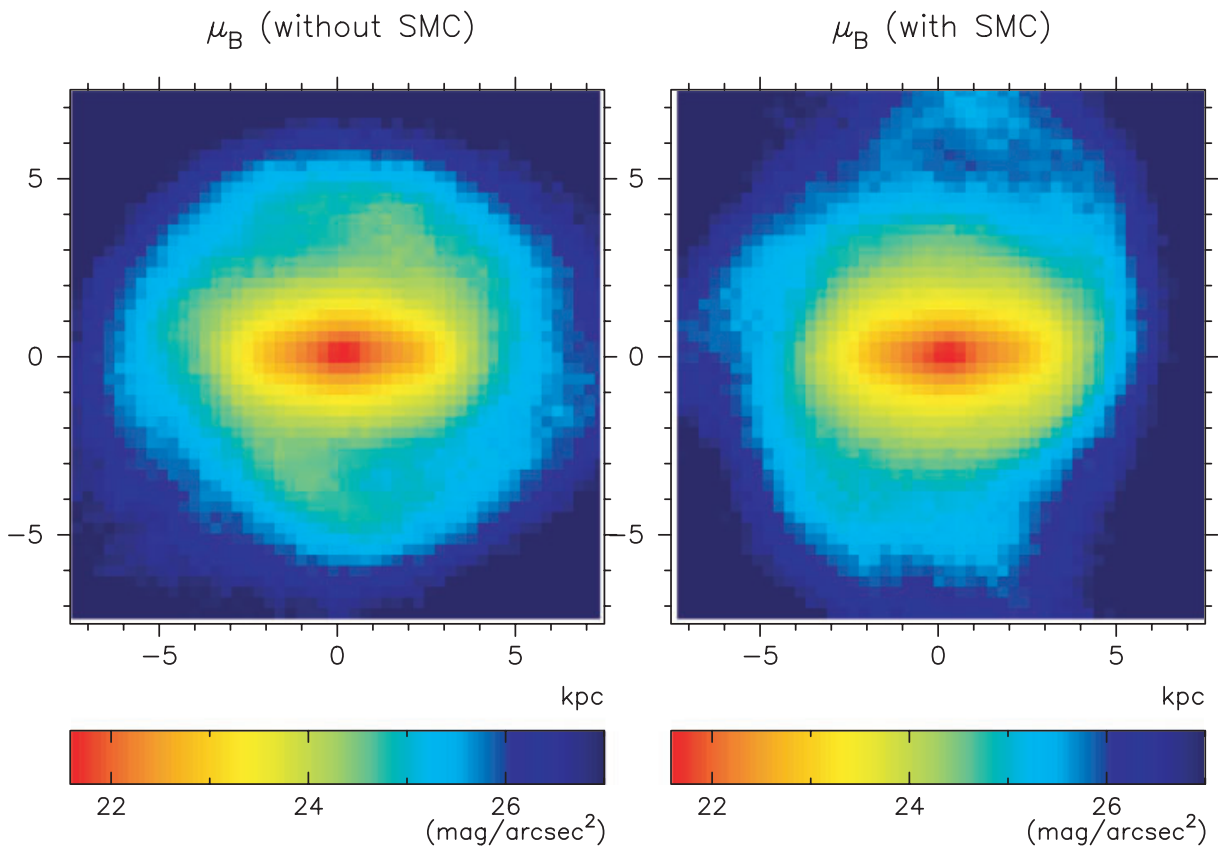

Figure 3 The 2D distributions of the $B$-band surface brightness ( $\left.\mu_{\mathrm{B}}\right)$ of the LMC at $T=0$ Gyr projected onto the $x-z$ plane (similar to the face-on view of the LMC) for the SMC-less model (left) and the standard model with the SMC (right). Here coordinate transformation is made so that the results can be more readily compared with the observations. The center of the bar is set to be coincident with the center of the frame for each model. The 2D map consists of 2500 cells with the cell size of $0.3 \mathrm{kpc}$ and $\mu_{\mathrm{B}}$ is estimated for each cell. Gaussian smoothing is used in order to derive smoothed $\mu_{\mathrm{B}}$ fields. Note that although the SMC-less model shows a elliptic disk, the center of the stellar bar in the disk does not appear to be coincident with the center of the outermost isophoto of the disk. Note also that the disk in the standard model appears to have a off-center bar.

Owing to mass-transfer from the SMC into the LMC during the Magellanic interaction, about $8 \%$ of the LMC's gaseous componets at $T=0 \mathrm{Gyr}$ were initially within the SMC. This transferred gas might well form either the diffuse halo gas around the LMC (Staveley-Smith et al. 2003) or kinematically odd components such as the 'L-component' (Luks \& Rohlfs 1992). Only 2\% of old stellar components of the SMC can be transferred into the central $7.5 \mathrm{kpc}$ of the LMC. Old stars and new stars formed from gas transferred from the SMC can be discussed in terms of structurally and kinematically odd stellar components of the LMC (e.g. Subramaniam \& Prabhu 2005).

It should be stressed here that the simulated gas distributions of the LMC do not show remarkable lack of gas within the stellar bar: observations clearly show HI deficiency within the off-center stellar bar (e.g. StaveleySmith et al. 2003). A possible reasons for the apparent failure of the models is that the present simulations can not model so realistically the conversion from Hi to molecular clouds and to field stars and star clusters in the central region of the LMC: star clusters and molecular gas are observed in the bar region of the LMC.

Figure 5 shows that the 2D distribution $\left(\mu_{\mathrm{s}}\right)$ of young stars with ages $\left(t_{\text {age }}\right)$ less than $20 \mathrm{Myr}$ is quite irregular and patchy, in particular in the left half of the disk. The highdensity regions of young stars can be seen within the stellar bar, where gas can be compressed into new stars owing to dynamical action of the bar. These irregular distributions of new stars can not be clearly seen in the $\mu_{\mathrm{s}}$ distribution for all new stars with $t_{\mathrm{age}}<200 \mathrm{Myr}$, which suggests that

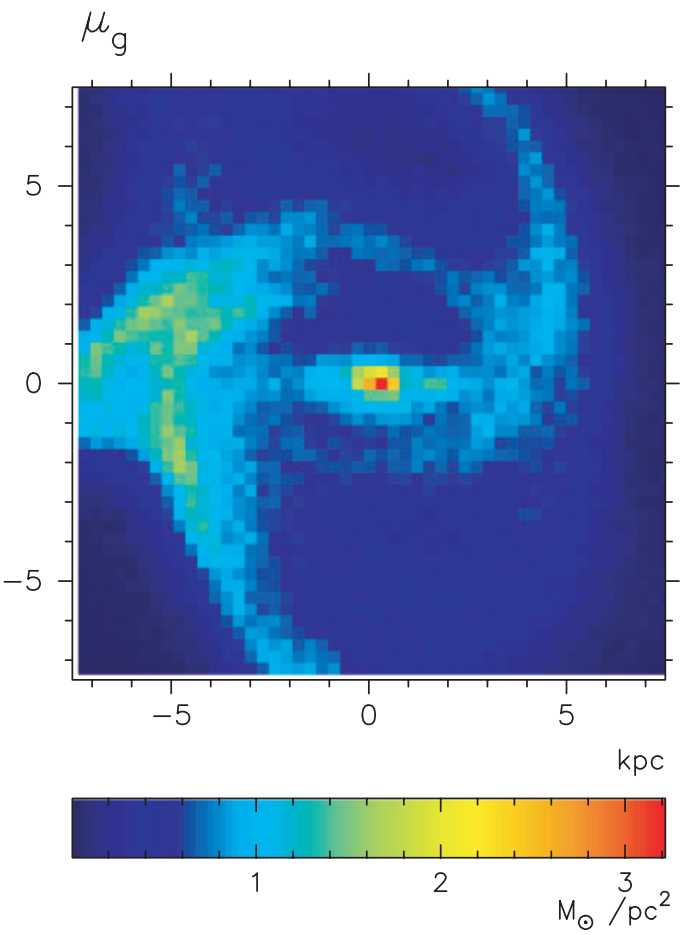

Figure 4 The 2D distribution of the surface gas density $\left(\mu_{\mathrm{g}}\right)$ of the LMC at $T=0 \mathrm{Gyr}$ projected onto the $x-z$ plane for the standard model. This figure is a magnified version of the last panel in Figure 2.

the LMC collision is responsible only for the asymmetric distribution of very young stars $\left(t_{\text {age }}<20 \mathrm{Myr}\right)$. The location of a clumpy distribution just above the left end of the bar (i.e. $(X, Z) \approx(-5,2) \mathrm{kpc}$ ) is coincident with 


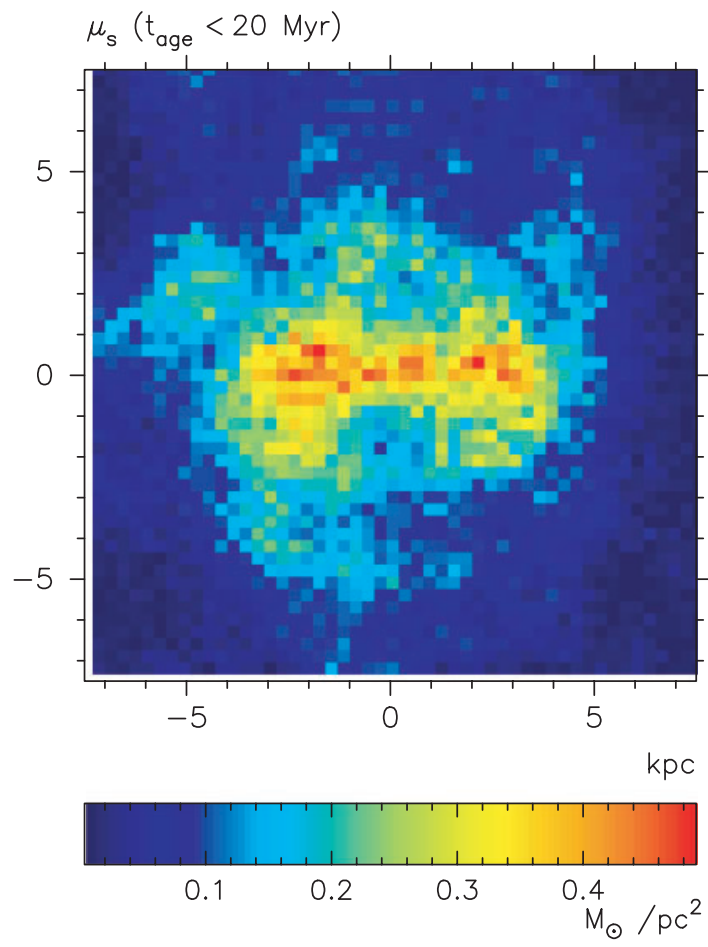

Figure 5 The 2D distribution of the stellar surface density $\left(\mu_{\mathrm{s}}\right)$ of the LMC at $T=0 \mathrm{Gyr}$ projected onto the $x-z$ plane for the standard model. Here the distribution is derived for very young stars with the ages $\left(t_{\text {age }}\right)$ less than $20 \mathrm{Myr}$. Note that there is an interesting peak just above the left edge (or eastern edge) of the bar.

the high-density region of the gaseous arm connected to the MB. This result is discussed in terms of 30 Doradus formation later in this paper.

The star formation rate averaged over the entire region of the LMC in the standard model is sharply increased by a factor of 6 at $T=-0.2 \mathrm{Gyr}$ in comparison with the isolated model with the star formation rate being almost constant $0.5 \mathrm{M}_{\odot} \mathrm{yr}^{-1}$ during the last $0.8 \mathrm{Gyr}$ evolution. Since previous (and the present study) showed that the SMC shows a burst of star formation about $0.2 \mathrm{Gyr}$ ago in their simulations (Yoshizawa \& Noguchi 2003), the sharp burst of star formation derived above for the LMC implies that (moderately strong) bursts of star formation can be synchronized in the LMC and the SMC about $0.2 \mathrm{Gyr}$ ago. This synchronized burst of star formation in the Clouds through the LMC-SMC tidal interaction can be proved by the observed age distributions of young star clusters in the Clouds (e.g. Girardi et al. 1995).

It should be noted here that Harris \& Zaritsky (2004) and Zaritsky \& Harris (2004) found a peak in the SMC's star formation history that coincided with close passage to the Milky Way. They, however, were unable to conclude whether the LMC-SMC interaction has had any noticeable effect on the SMC's star formation history. Therefore, the simulated burst of star formation about $0.2 \mathrm{Gyr}$ in the SMC has not been observationally confirmed yet.

We confirm that the Clouds can merge with each other to form a single Magellanic cloud within several Gyr from the present $(T=0 \mathrm{Gyr})$ in some models, because the SMC

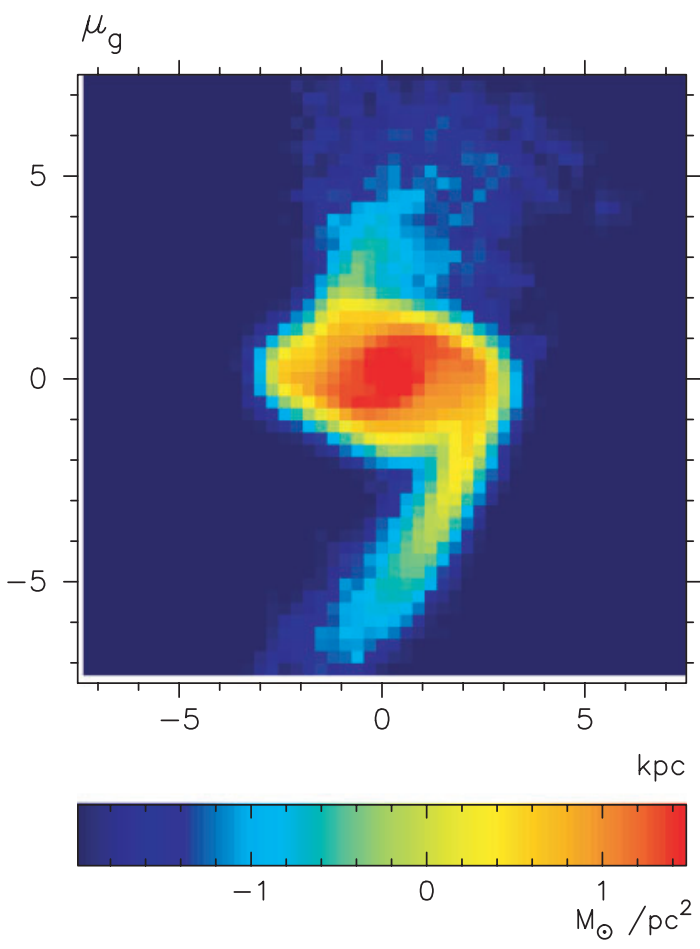

Figure 6 The projected distribution of smoothed (column) gas densities of the SMC about $0.14 \mathrm{Gyr}$ ago (i.e. $60 \mathrm{Myr}$ after the last Magellanic interaction). The smoothed densities $\left(\mu_{\mathrm{g}}\right)$ are give in a logarithmic scale. The lower tidal tail with a higher gas density is the forming MB.

continues to sink into the LMC due to dynamical friction between the LMC's halo and the SMC. The LMC is transformed into a disk galaxy with a S0-like morphology, a bar, a thick stellar disk and rotational kinematics during merging. The newly formed 'Magellanic Cloud' also has a stellar halo formed mainly from the tidal debris of the SMC and accordingly the expected mean metallicity of the halo is as high as $[\mathrm{Fe} / \mathrm{H}] \sim-0.7$, which is significantly higher than that of the Galactic old stellar halo $([\mathrm{Fe} / \mathrm{H}] \sim-1.6)$. These results will be discussed in our forthcoming papers in terms of the origin of stellar halos in S0 galaxies.

\subsection{The Magellanic Bridge}

The outer part of the SMC's gas disk is strongly stretched during the LMC-SMC interaction to form two tidal tails, one of which is observed as the MB. Since the Hi gas disk is initially larger than the stellar one in the SMC model with $R_{\mathrm{g}} / R_{\mathrm{S}}=2$, the $\mathrm{MB}$ is composed of metal-poor gas and stars formed from the gas (i.e. almost no old stars originating from the inner stellar disk in the MB). About $25 \%$ of the initial gas of the SMC is stripped to form the $\mathrm{MB}$, which means that the total mass in the MB is about $10^{8} \mathrm{M}_{\odot}$ for the SMC model with the gas mass fraction of 0.5 . The final gas mass in the $\mathrm{MB}$ depends on $R_{\mathrm{g}} / R_{\mathrm{S}}$ of the SMC in the sense that a larger amount of gas can be distributed in the MB for the models with larger $R_{\mathrm{g}} / R_{\mathrm{s}}$.

The LMC-SMC tidal interaction can also significantly change the recent star formation histories, not only in the central region of the SMC's gas disk, but also in its outer 


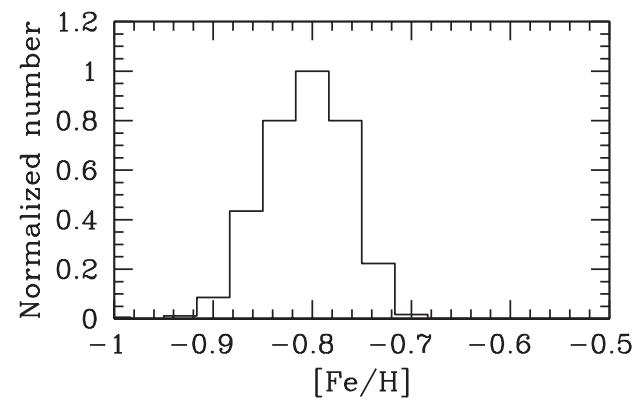

Figure 7 The metallicity distribution function of new stars formed in the MB (normalized to the maximum number of stars in the metallicity bins). Note that the peak metallicity is around $[\mathrm{Fe} / \mathrm{H}]=-0.8$, which is significantly smaller than the central stellar metallicity of the simulated SMC.

part (Yoshizawa \& Noguchi 2003). Figure 6 shows that the SMC's outer gas disk is strongly compressed by the interaction so that gas densities along the forming $\mathrm{MB}$ can exceed the threshold gas density of star formation (i.e. $3 \mathrm{M}_{\odot} \mathrm{pc}^{-2}$ ). New stars, therefore, can form in the MB and some of the stars are finally stripped to become interCloud stars with young ages of less than 200 Myrs. The spatial distribution of these simulated inter-Cloud stars can be compared with that of blue stars observed in the interCloud region (e.g. Irwin et al. 1990; Rolleston et al. 1999).

Since the MB is formed from the outer gas, where the metallicity is significantly smaller owing to the negative metallicity gradient of the SMC, the metallicities of young stars in the MB can be significantly small: the metal-poor stars are due to the adopted metallicity gradient of the SMC's gas disk (not due to the dynamical mixing during the interaction). Figure 7 shows that the metallicity distribution of new stars in the MB has a peak of $[\mathrm{Fe} / \mathrm{H}] \sim-0.8$, which is about 0.2 dex smaller than the central stellar metallicity of the simulated SMC. Although this result is consistent with the presence of young, metal-poor stars in the $\mathrm{MB}$, the observed stars with metallicities 0.5 dex lower than those of SMC (i.e. corresponding to $[\mathrm{Fe} / \mathrm{H}] \sim-1.1$ ) cannot be well reproduced. Only $0.1 \%$ of the gas mass of the SMC is converted into new stars in the MB, which means that the mass ratio of new stars to gas in the MB is $\sim 0.004$. The present model thus provides a physical explanation for the origin of the observed formation sites of new stars along the MB (Mizuno et al. 2006).

Recently Harris (2007) has reported possible evidence for the MB without old field stars and has suggested that the MB was formed from purely gaseous components. The present models, however, are not consistent with this result, in the sense that a minor fraction of old disk stars can be stripped tidally from the SMC and still lie within the $\mathrm{MB}$ at the present time. We will discuss this problem in a more extensive and quantitative way in our future papers.

\section{Discussions}

\subsection{Formation of 30 Doradus}

30 Doradus and young clusters within and close to it have been extensively discussed both theoretically and observationally in the contexts of triggered star formation by stellar feedback effects and initial mass functions (IMFs) of star clusters (e.g. Westerlund 1997; Selman et al. 1999; van den Bergh 2000). However it remains unclear how giant molecular clouds can be converted into massive new clusters embedded in the HII region of 30 Doradus in the evolution of the LMC. In particular, it is unclear how galaxy-scale dynamics is important for the formation of massive gas clouds that are progenitors of 30 Doradus. We here consider that the location of 30 Doradus with respect to HI gas and molecular gas provides a vital clue to the formation process of 30 Doradus.

30 Doradus, with the central super-cluster R136, is located just above the eastern end of the LMC's bar (e.g. van den Bergh 2000) and the peculiar Hi arms appear to emerge from above the eastern end of the bar (see Staveley-Smith et al. 2003, Figure 1). Large scale mapping of molecular gas in the vicinity of 30 Doradus in the LMC has recently discovered a strong concentration of giant molecular clouds (GMCs) close to 30 Doradus (Ott et al. 2007). Interestingly, the present simulations also show that both gaseous arms with high densities and relatively densely populated regions of very young stars $\left(t_{\text {age }}<20 \mathrm{Myr}\right)$ can be seen above the left side of the LMC's bar (corresponding to the eastern edge of the bar). This amazing coincidence between the observations and the simulations implies that the origin of 30 Doradus (and thus R136) could result from dynamical and hydrodynamical interaction between the Clouds in the last Magellanic interaction.

Thus the preset simulations suggest that the observed unique location of 30 Doradus tells us about its formation process: 30 Doradus originates from GMCs that were developed in high-density regions of asymmetric gaseous spiral arms formed in the last Magellanic interaction. Since the dynamical influences of galaxy interaction can last for one rotation period (corresponding to $0.25 \mathrm{Gyr}$ for the LMC), the formation of star clusters with ages less than 0.25 Gyr in the LMC might well be triggered by the Magellanic interaction. We accordingly suggest that 30 Doradus is just one example of many star-forming regions triggered by the interaction with the SMC (and the Galaxy) and the resultant large-scale dynamical effects inside the LMC.

The distribution of star-forming regions like 30 Doradus is observed to be asymmetric, irregular, and patchy in the LMC. The origin of such a distribution of star-forming regions and young massive stars has long been discussed in terms of different physical mechanisms, such as stochastic self-propagating star formation (SSPSF, Feitzinger et al. 1981), gas compression in a bow shock due to the Galactic warm gas (de Boer et al. 1998) and dynamical perturbation of the LMC's off-center bar (Gardiner et al. 1998). The simulated asymmetric distribution of young stars in the LMC disk suggests that the Magellanic collision can also play a role in forming asymmetric distributions of star-forming regions and young stars. It is thus our future study to understand the relative importance of these mechanisms (e.g. SSPSF and the 
Magellanic collision) in the formation of the asymmetric distributions in the LMC.

\subsection{Off-Center Bars and Dynamical Influences of Dark Matter Subhalos}

Although barred Magellanic-type dwarfs that appear to be pairs of galaxies are not rare (e.g. Freeman 1984), the existence of off-center bars is seen in systems that are not obviously interacting (Vaucouleurs \& Freeman 1972; Gardiner et al. 1998). Recent cosmological numerical simulations (e.g. Susa \& Umemura 2004) have demonstrated that star formation in low-mass systems with masses of $\sim 10^{8} \mathrm{M}_{\odot}$ can be severely suppressed by cosmic reionization so that these systems can become galaxies that are not optically observable (dark galaxies). We accordingly suggest that the formation of off-center bars in Magellanic-type dwarfs with apparently no optical companions could possibly be due to the unequal-mass galaxy collision between them and their invisible companions. The presence of off-center bars can be useful for proving missing satellites through interaction with them.

\subsection{Origin of ESO 121-SCO3}

The LMC has a unique cluster formation history in that nearly all of its GCs were formed either $\sim 13 \mathrm{Gyr}$ ago or less than 3 Gyr ago (Da Costa 1991). Recently Mackey, Payne \& Gilmore (2006) have revealed that the GC ESO $121-\mathrm{SC} 03$ in the $\mathrm{LMC}$ has $[\mathrm{Fe} / \mathrm{H}]=-0.94 \pm 0.10$ and an age range of $8.3-9.8 \mathrm{Gyr}$ and have thus confirmed that ESO 121-SC03 is the only known cluster to lie squarely within the LMC age gap (see also Mateo, Hodge \& Schommer 1986; Geisler et al. 1997) Previous numerical simulations showed that the first close encounter between the LMC and the SMC about 3-4 Gyr ago was the beginning of a period of strong tidal interaction that likely induced dramatic gas cloud collisions, leading to an enhancement in the formation of globular clusters that has been sustained by strong tidal interactions to the present day (Bekki et al. 2004b). They thus suggested that the origin of the age gap is closely associated with this dynamical coupling between the LMC and the SMC about 3-4 Gyr ago. However no theoretical studies have explained why ESO 121-SC03 is the only GC that was formed between 3 and $13 \mathrm{Gyr}$ in the history of the LMC.

The simulated small yet non-negligible amount of the SMC's halo and old stellar components transferred into the LMC implies that some of the SMC's old clusters, which are observed to be significantly younger than the LMC's counterparts (e.g. Piatti et al. 2005), can be transferred into the LMC's halo and thus identified later as one of the LMC's GCs. The pericenter distance of the LMC-SMC orbit about 0.2 Gyr is much smaller than that ( $\sim 20 \mathrm{kpc}$, which is larger than the tidal radius of the SMC) about $1.5 \mathrm{Gyr}$ ago when the Magellanic Stream was formed during the pericenter passage of the SMC (GN95). It is therefore likely that GCs of the SMC could have been transferred into the LMC not $1.5 \mathrm{Gyr}$ ago but
0.2 Gyr ago. We accordingly suggest that the ESO 121SC03 came from the SMC about 0.2 Gyr ago during the last Magellanic interaction.

Previous simulations (e.g. Weinberg 2000; BC05) suggest that the origin of the stellar halo of the LMC is closely associated with the tidal interaction between the LMC and the Galaxy (and the SMC). Recent observations on kinematics of carbon stars in the entire region of the LMC have suggested that the LMC's stellar components are strongly influenced by the interaction (e.g. Olsen \& Massey 2007). Thus the formation process of the halo GC (ESO 121SC03) suggested in the present paper can be in a striking contrast to that of the stellar halo in the LMC.

\subsection{Giant HI Holes in the LMC}

Although it is well known that gas-rich dwarf irregular galaxies (e.g. LMC, SMC, HoII, and DDO 50) have giant $\mathrm{HI}$ holes in their interstellar medium, it remains unclear which physical mechanisms are responsible for the formation of giant Hi holes in these galaxies (Rhode et al. 1999). One physical mechanism for the formation of giant HI holes (and surrounding peculiar stellar associations) is considered to be the strong impact of high-velocity clouds onto gas disks in these galaxies (see Efremov 2004 for detailed discussions). Although this Hi impact model can be the most natural explanation for the LMC's Hi holes with no optical counterparts (e.g. star clusters that can form holes from the energetic stellar winds of massive stars, Efremov 2004), it is unclear how such high-velocity impact of Hi gas clouds can happen in the gas disk of the LMC.

The present simulations show that only a small fraction of gas (up to 8\%) initially in the SMC can be transferred into the LMC's gas disk during the LMC-SMC interaction. We thus propose the following scenario for the formation of giant $\mathrm{HI}$ holes in the LMC. Owing to the large relative velocity of the Clouds $\left(>60 \mathrm{~km} \mathrm{~s}^{-1}\right)$, the SMC's gas clouds can collide with the LMC's gas disk with a relative velocity higher than the sound velocities of the gas $\left(\sim 4 \mathrm{~km} \mathrm{~s}^{-1}\right)$. These collisions can induce heating of gas in the shocked gas layers to cause ionization of Hi gas in the LMC. The locations where SMC's Hi gas collide with the LMC (thus where Hi gas is ionized) can be observed as giant $\mathrm{HI}$ holes in the LMC. The present simulations do not have enough resolution (i.e. sub-pc scale) to investigate these processes during the LMC-SMC interaction. It is thus our future intention to investigate whether $\mathrm{HI}$ holes in the LMC can be formed from the high velocity impact of the SMC's gas clouds based on more sophisticated, high-resolution hydrodynamical simulations of the LMC-SMC interaction.

\section{Conclusions}

We have numerically investigated stellar and gaseous distributions and recent star formation histories of the LMC and the SMC, as they interact with each other and with the Galaxy. We summarize our principle results as follows. 
The last dynamical and hydrodynamical interaction between the Clouds, about $\sim 0.2 \mathrm{Gyr}$ ago, could have formed the apparently off-center bar and peculiar $\mathrm{HI}_{\mathrm{I}}$ spirals of the LMC. The location and the morphology of the simulated spiral in the southern part of the LMC is similar to those of the 'molecular ridge' observed in the LMC (e.g. Fukui et al. 1999; Kawamura et al. 2007).

The present spatial distributions of young stars with ages less than $\sim 20 \mathrm{Myr}$ in the LMC can be significantly asymmetric and clumpy owing to the interaction between the Clouds and the Galaxy. The simulated new stars show a strong concentration along the LMC's stellar bar, which is composed of old stars. These results suggest that the observed irregular and clumpy distributions of young stars and star-forming regions (e.g. HII regions) in the LMC are due to the last Magellanic interaction.

A small but non-negligible fraction of stellar and gaseous components can be transferred from the SMC into the LMC during the interaction to form diffuse halo components around the LMC. This result implies that some fraction of GCs in the SMC formed before the last Magellanic interaction could have been transferred into the LMC. We have suggested that the LMC's GC ESO 121-SC03, which falls into the age gap from 3 to $13 \mathrm{Gyr}$, originates from the SMC.

The outer part of the SMC's gas disk can be strongly compressed by the tidal force from the LMC and the Galaxy and, finally, stretched to form tidal tails, one of which can be observed as the MB. During the formation of the $\mathrm{MB}$, the gas density along the forming MB exceeds the threshold gas density of star formation, so that new stars can be formed in the MB. Star formation by tidal compression can explain why young stars and molecular clouds can be observed in the MB (e.g. Mizuno et al. 2006).

The metallicity distribution function of new stars in the MB has a peak of $[\mathrm{Fe} / \mathrm{H}] \sim-0.8$, which is significantly smaller than the central stellar metallicity of the simulated SMC $([\mathrm{Fe} / \mathrm{H}] \sim-0.6)$. Although the present model explains the presence of young, metal-poor stars in the $\mathrm{MB}$, it is still unclear why some of the inter-Cloud stars have metallicities as low as $[\mathrm{Fe} / \mathrm{H}] \sim-1.1$ (e.g. Rolleston et al. 1999).

\section{Acknowledgments}

We are grateful to the anonymous referee for valuable comments, which helped to improve the present paper. The numerical simulations reported here were carried out on GRAPE systems kindly made available by the Astronomical Data Analysis Center (ADAC) at National Astronomical Observatory of Japan (NAOJ). K.B. acknowledges financial support from the Australian Research Council (ARC) and ADAC throughout the course of this work.

\section{References}

Bekki, K. \& Chiba, M., 2005, MNRAS, 356, 680

Bekki, K. \& Chiba, M., 2006, ApJ, 637, L97

Bekki, K., Beasley, M. A., Forbes, D. A. \& Couch, W. J., 2004a, ApJ, 602,730
Bekki, K., Couch, W. J., Beasley, M. A., Forbes, D. A., Chiba, M. \& Da Costa, G., 2004b, ApJ, 610, L93

Broeils, A. H. \& van Woerden, H., 1994, A\&AS, 107, 129

Da Costa, G. S., 1991, in IAU Symp., 148, The Magellanic Clouds, Eds. Haynes, R. \& Milne, D. (Dordrecht: Kluwer), 183

de Boer, K. S., Braun, J. M., Vallenari, A. \& Mebold, U., 1998, A\&A, 329, L49

de Vaucouleurs, G. \& Freeman, K. C., 1972, VA, 14, 163

Efremov, Y. N., 2004, ASP Conf. Ser., 316, Order and Chaos in Stellar and Planetary Systems, Eds. Byrd, G., Kholshevnikov, K., Myllri, A., Nikiforov, I. \& Orlov, V. (San Francisco: Astronomical Society of the Pacific), 261

Feitzinger, V., Glassgold, A. E., Gerola, H. \& Seiden, P. E., 1981, A\&A, 98, 371

Freeman, K. C., 1984, in IAU Symp., 108, Structure and Evolution of the Magellanic Clouds, Eds. van den Bergh, S. \& Boer, K. S. D. (Dordrecht: Reidel), 107

Fujimoto, M. \& Kumai, Y., 1997, AJ, 113, 249

Fukui, Y., et al., 1999, PASJ, 51, 745

Gardiner, L. T. \& Noguchi, M., 1996, MNRAS, 278, 191

Gardiner, L. T., Turfus, C. \& Putman, M. E., 1998, ApJ, 507, L35

Geisler, D., Bica, E., Dottori, H., Clari, J. J., Piatti, A. E. \& Santos Jr., J. F. C., 1997, AJ, 114, 1920

Girardi, L., Chiosi, C., Bertelli, G. \& Bressan, A., 1995, A\&A, 298, 877

Harris, J., 2007, ApJ, in press (astro-ph/0612107)

Harris, J. \& Zaritsky, D., 2004, AJ, 127, 1531

Irwin, M. J., Demers, S. \& Kunkel, W. E., 1990, AJ, 99, 191

Kallivayalil, N., van der Marel, R. P. \& Alcock, C., 2006, ApJ, 652, 1213

Kawamura, A., et al., 2007, in preparation

Kennicutt Jr., R. C., 1998, ARA\&A, 36, 189

Kunkel, W. E., Demers, S., Irwin, M. J. \& Albert, L., 1997, ApJ, 488, L129

Luks, Th. \& Rohlfs, K., 1992, A\&A, 263, 41

Mackey, A. D., Payne, M. J. \& Gilmore, G. F., 2006, MNRAS, 369, 921

Mateo, M., Hodge, P. \& Schommer, R. A., 1986, ApJ, 311, 113

Mizuno, N., Muller, E., Maeda, H., Kawamura, A., Minamidani, T., Onishi, T., Mizuno, A. \& Fukui, Y., 2006, ApJ, 643, L107

Murai, T. \& Fujimoto, M., 1980, PASJ, 32, 581

Olsen, K. A. G. \& Massey, P., 2007, ApJ, 656, L61

Ott, J., et al., 2007, in preparation

Piatti, A. E., Sarajedini, A., Geisler, D., Seguel, J. \& Clark, D., 2005, MNRAS, 358, 1215

Rhode, K. L., Salzer, J. J., Westpfahl, D. J. \& Radice, L. A., 1999, AJ, 118, 323

Rolleston, W. R. J., Dufton, P. L., McErlean, N. D. \& Venn, K. A., 1999, A\&A, 348, 728

Selman, F., Melnick, J., Bosch, G. \& Terlevich, R., 1999, A\&A, 347, 532

Staveley-Smith, L., Kim, S., Calabretta, M. R., Haynes, R. F. \& Kesteven, M. J., 2003, MNRAS, 339, 87

Subramaniam, A. \& Prabhu, T. P., 2005, ApJ, 625, L47

Susa, H. \& Umemura, M., 2004, ApJ, 610, L5

van den Bergh, S., 2000, The Galaxies of the Local Group (Cambridge: Cambridge University Press)

van der Marel, R. P., 2001, AJ, 122, 1827

Vazdekis, A., Casuso, E., Peletier, R. F. \& Beckman, J. E., 1996, ApJS, 106, 307

Yoshizawa, A. \& Noguchi, M., 2003, MNRAS, 339, 1135

Weinberg, M. D., 2000, ApJ, 532, 922

Westerlund, B. E., 1997, The Magellanic Clouds (Cambridge: Cambridge University Press)

Zaritsky, D. \& Harris, J., 2004, ApJ, 604, 167 